\title{
Fermi-Liquid-Type Spectral Function AND ANgle Resolved Photoelectron Spectra of the $\mathrm{Ti}-3 \mathrm{~d}_{z^{2}}$-BAND OF $\mathrm{TiTe}_{2}$
}

\author{
S. Harm, R. Dürig, R. Manzke, M. Skibowski \\ Institut für Experimentalphysik, Universität Kiel, D-24118 Kiel, Germany \\ R. Claessen \\ Fachrichtung Experimentalphysik, Universität des Saarlandes, D-66041 Saarbrücken, Germany \\ J. W. Allen \\ Randall Laboratory, University of Michigan, Ann Arbor, Michigan 48109-1120
}

\begin{abstract}
Angle and temperature dependent photoelectron spectra with high energy and momentum resolution $\left(\Delta E=30 \mathrm{meV}, \Delta \theta< \pm 0.5^{\circ}\right)$ were taken near the Fermi energy $\left(E_{\mathrm{b}}<1 \mathrm{eV}\right)$ of the quasi-two-dimensional, metallic layer compound $\mathrm{TiTe}_{2}$ along the direction $\overline{\Gamma M}$. The aim was to test the range of the validity of the Fermi liquid model using a. Taylor expansion for the self-energy used by Claessen et al. [1] for a system whose low energy properties are those of a normal metal. Single crystalline samples have been prepared by vapor growth technique using different amounts of $\mathrm{Te}$ in order to influence the intercalation of surplus $\mathrm{Ti}$. We found a dependence of the dispersion of the Ti-3 $\mathrm{d}_{z^{2}}$-derived band on the stoichiometry of the samples. For an exact line shape analysis the spectra were fitted by a least-squares-method to a Fermi-liquid-type spectral function taking into account the crucial effects due to the apparatus, as there are the energy resolution and finite angle resolution which leads to a limited integration in $\mathrm{k}$-space. In the region where the Ti-3 $\mathrm{d}_{z^{2}}$-derived band crosses the Fermi level we found excellent agreement between the experimental line shape and the Fermi liquid model. On the other hand there are distinct deviations between the theoretical and the experimental line shape at the Brillouin zone boundary even for a self-energy proposed by Matho [2] without Taylor expansion.
\end{abstract}

\section{Introduction}

Angle Resolved Photoemission Spectroscopy (ARPES) is a powerful tool to investigate the occupied band structure of condensed matter. For this purpose usually only the energy positions of the emission maxima in the experimental spectra are used. However, much information of the photoemission spectra - especially the line shape of the peaks - are ignored, if only the band structure is derived.

In this work we deal with an already well known system 1T-TiTe 2 [3] in order to test the application of line shape analysis to photoemission spcetra in a rclativcly simplc casc. The results of such an analysis may give a deeper insight in many particle interactions (such as electronelectron correlation etc.). Further applications of this method could also be of great interest in the study of HTSC's. Performing a line shape analysis we have to distinguish between intrinsic effects of the sample and effects due to the apparatus. Only if we have a good knowledge of the latter effects a line shape analysis can give us correct insight into physical processes in the sample. Additionally a well defined spectrum of the sample is required which should contain if possible clearly identified single peaks with neglegible 
inelastic background. Finally a theoretical based model-function describing the measured feature is necessary.

The two-dimensional layered transition metal dichalcogenide $\mathrm{TiTe}_{2}$ satisfies the mentioned conditions. We examined the strong emission of

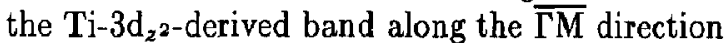
which crosses the Fermi energy and fully disappears for angles $\theta<12^{\circ}$. The behaviour of this peak near the Fermi energy depends weakly, but noticeably on the stoichiometry of the investigated sample. We carried out several measurements that showed this.

$\mathrm{TiTe}_{2}$ differs from the other transition metal dichalchogenides because no CDW/PLD induced phase transitions occur. Moreover the clear separation of $\mathrm{d}$ - and $\mathrm{p}$-bands allows a line shape analysis of the Ti-3 $\mathrm{d}_{z^{2}}$-peak. Regarding resistivity measurements [4] the conduction electrons of $\mathrm{TiTe}_{2}$ show the same behaviour as found for quasiparticle excitations combined with phonons in a normal metal. The temperature dependence of resistivity is linear in the range from room temperature down to $60 \mathrm{~K}$. Between $60 \mathrm{~K}$ and $10 \mathrm{~K}$ there is a dependence like $\propto \mathrm{T}^{5}$ as expected for electron-phonon scattering in a Fermi liquid.

\section{Theoretical Aspects}

Within the usually applied three step model of photoemission and using the sudden approximation, the ARPES signal is proportional to the product of the transition malrix element, the spectral function $A(\vec{k}, \omega)$, and the Fermi-Diracdistribution $f_{T}(\omega)$. However, going beyond this simple description there are additional complications that might affect the photoemission line shape. For example, in the more comprehensive one-step theory it is shown that the lifetime of the photoelectron adds to the total ARPES linewidth. Since the final-state energy width is mixed in with a weight factor $v_{h \perp} / v_{e \perp}$, where $v_{h \perp}$ and $v_{e \perp}$ denote the band velocities of the photohole and the photoelectron perpendicular to the surface, the effect of the final electron state broadening is sufficiently suppressed for quasitwo-dimensional systems, where $v_{h \perp} \ll v_{e \perp}$ [5]. That is the reason why detailed line shape studies in the way mentioned above can best be per- formed on layered systems like $\mathrm{TiTe}_{2}$ or HTSC's or - provided that the intensity is high enough - on surface states. Finally scattering processes at surface imperfections and diffraction of the outgoing photoelectrons can influence the photoemission spectrum and its line shape. Only if all these mentioned effects are small enough the ARPES signal can be taken to be representative of the electron removal spectrum.

The spectral function $\mathbf{A}(\vec{k}, \omega)$ itself is given by

$$
A(\vec{k}, \omega)=\frac{1}{\pi} \operatorname{Im}(G(\vec{k}, \omega))
$$

$G(\vec{k}, \omega)$ is the Green's function of the interacting electron system. The one-particle Green's function is given by

$$
G(\vec{k}, \omega)=\frac{1}{\omega-\epsilon_{k}-\Sigma(\vec{k}, \omega)}
$$

where $\epsilon_{k}$ denotes the free-particle energy of the non interacting system. $\Sigma(\vec{k}, \omega)$ describes the complex self-energy containing all contributions due to electron-electron interactions. In this paper we describe the experimental consequences for two different types of self-energies. For a three-dimensional Fermi liquid one normally finds a 2nd-order Taylor expansion for the $\vec{k}$ independent self-energy [6] in the form

$$
\Sigma(\omega)=\alpha \omega+i \beta \omega^{2}
$$

Using this self-energy the Fermi liquid spectral function is given by

$$
A(\vec{k}, \omega) \propto \frac{\beta^{\prime} \omega^{2}}{\left(\omega-\epsilon_{k}^{\prime}\right)^{2}-\beta^{\prime 2} \omega^{4}}
$$

with $\epsilon_{k}^{\prime}=\frac{1}{1-\alpha} \epsilon_{k}$ and $\beta^{\prime}=\frac{1}{1-\alpha} \beta$. Figure 1 shows this spectral function for various values of $c_{k}$. For $\epsilon_{k}=0$ a delta-peak indicating the infinite lifetime of quasiparticles at the Fermi surface is obtained. 


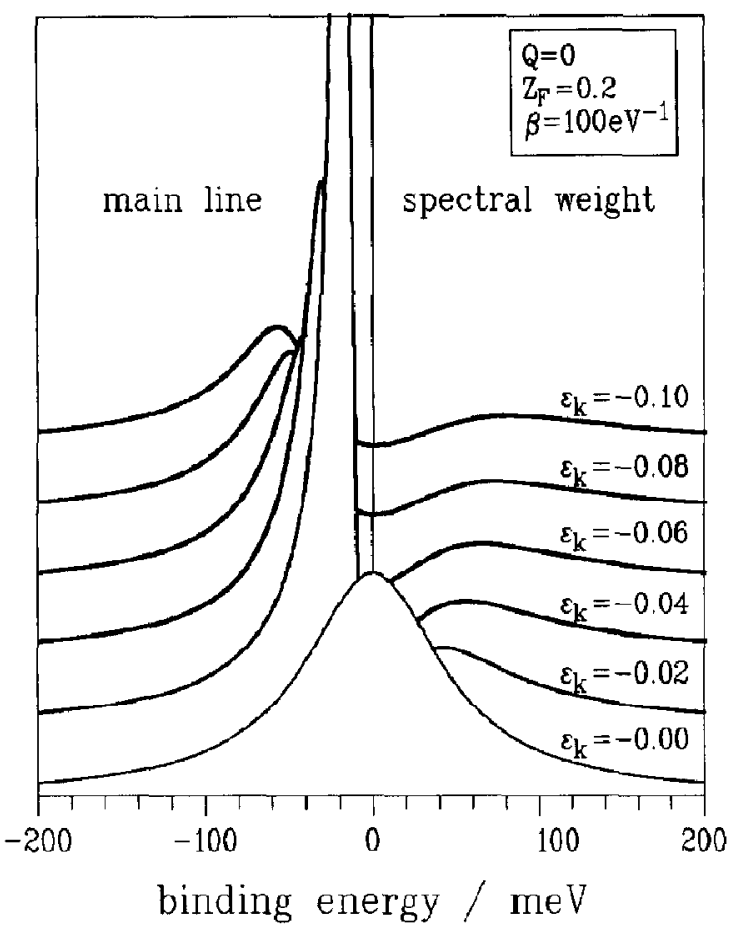

Fig. 1: Spectral-function of Fermi liquid model used by Claessen et al. [1].

However, since the approximation for the selfenergy is valid only for small values of $\omega$ the resulting spectral function is only correct in the direct neighbourhood of the Fermi energy. Especially for $\omega>1 / \beta^{\prime}$ Matho [2] shows in his comment on the work by Claessen et al. [1] that the used self-energy produces an incorrect propagator with a pole in the physical sheet leading to a violation of the fermionic sumrule. Matho points out that the Taylor expansion of the self-energy corresponds to a Green's function on the Fermi surface $\left(\vec{k}=\overrightarrow{k_{F}}\right)$ in the form

$$
G\left(\overrightarrow{k_{F}}, \omega\right)=\frac{Z_{F}}{\omega}+\frac{-Z_{F}}{\omega-\omega_{0}}
$$

with $Z_{F}=\frac{1}{1-\alpha}$ and $\omega_{0}=-\frac{i}{\beta^{\prime}}$. The two summands in this Green's function describe the quasiparticle peak and the elastic but incoherent background due to quasiparticle excitations, respectively. For the quasiparticle peak one obtains the correct weight $Z_{F}$ but the weight $-Z_{F}$ for the background is quite unphysical. For that reason Matho suggests a more correct propagator on the Fermi surface of the form

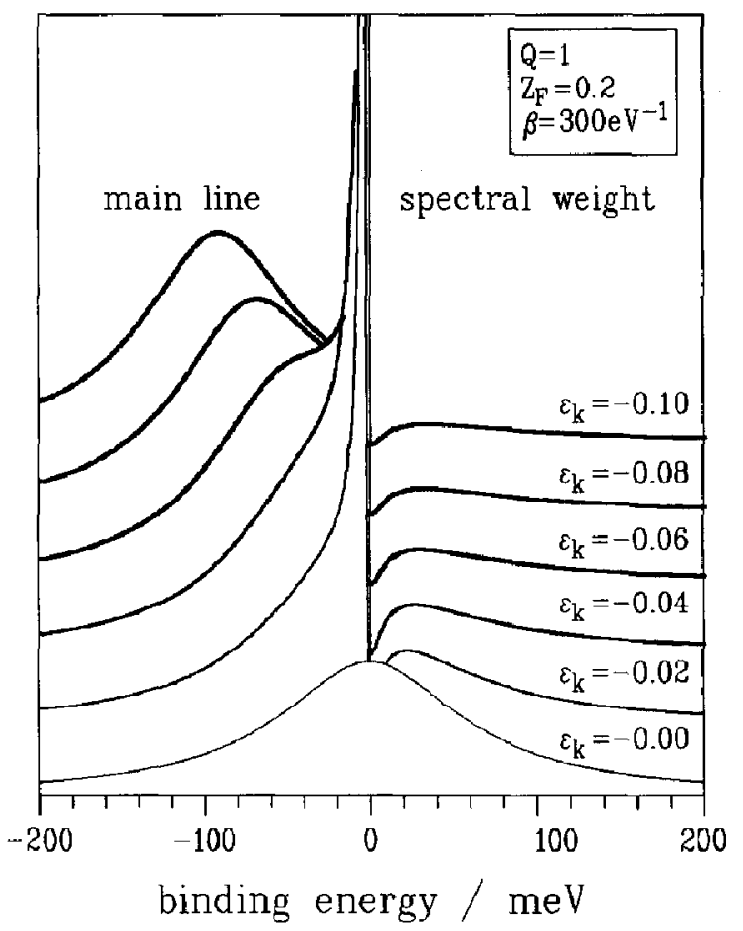

Fig. 2: Spectral-function of Fermi liquid model proposed by Malho [2].

$$
G\left(\overrightarrow{k_{F}}, \omega\right)=\frac{Z_{F}}{\omega}+\frac{\left(Q-Z_{F}\right)}{\left(\omega-\omega_{1}\right)}
$$

This propagator models the low energy spectrum of weight $Q$ (consisting of the quasiparticle weight $Z_{F}$ and the low-lying incoherent excitations), being separated from the high-energy incoherent excitations such as plasmon or Hubbard satellites which have to be taken into consideration by other additive terms in the propagator. The imaginary part of $\omega_{1}$ is given by

$$
\operatorname{Im}\left(\omega_{1}\right)=-\frac{Q-Z_{F}}{Z_{F}} \operatorname{Im}\left(\omega_{0}\right)=\frac{Q-Z_{F}}{\beta Z_{F}^{2}}
$$

The real part of $\omega_{1}$ shifts the energy position of the incoherent background. In this paper we deal with $Q=1$ and $\operatorname{Re}\left(\omega_{1}\right)=0$ only. Starting with the new Green's function on the Fermi surface one ubtains the self-energy fron the equation

$$
\Sigma(\omega)=\omega-\frac{1}{G\left(\vec{k}=\overrightarrow{k_{F}}, \omega\right)}
$$

Inserting this in (2) and (1) yields the spectral function $A(\vec{k}, \omega)$. The analytical form of 
$A(\vec{k}, \omega)$ in this case is rather complicated but it can be easily calculated numerically. Figure 2 shows in comparison to figure 1 the spectral function obtained for different values $\epsilon_{k}$ for the selfenergy proposed by Matho. For $\varepsilon_{k}=0$ there is again a delta-peak, but note the strong deviations of the line shape especially for higher values of the peak binding energy $\epsilon_{k}$. Beside the variation in the line shape the maximum of the spectral function lies at different energy positions when compared with figure 1 .

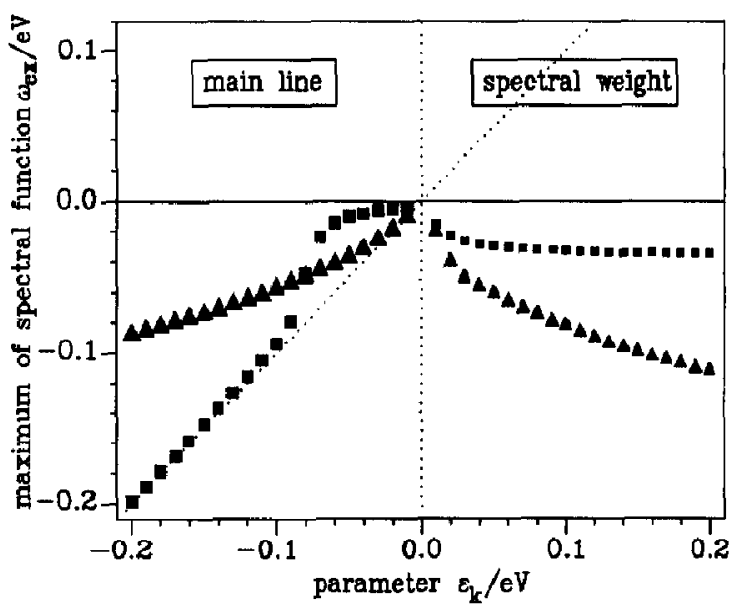

Fig. 3: Energy position of the maxima of the spectral function as function of the parameter $\epsilon_{k}$. The triangles mark the maxima for the Taylor-expanded self-energy, the maxima of the spectral function proposed by Matho are denoted by squares.

In figure 3 the maxima of the spectral function (Taylor expansion: triangles, Matho's self-energy: squares) are plotted as a function of the binding energy $\epsilon_{k}$. As indicated by the dashed line the position of the maxima of Matho's spectral function equals - as expected $-\epsilon_{k}$ for $\epsilon_{k} \leq-0.1 \mathrm{eV}$.

\section{Experimental}

Single crystals have been prepared using the vapor growth technique with iodine as transport agent. The pure substances are filled in quartz tubes together with an amount of iodine and an excess of Te. The tubes are exposed to an exactly fixed temperature gradient for several days yielding single crystal samples up to the dimensions about $5 \mathrm{~mm} \times 5 \mathrm{~mm}$. These samples then are mounted on a cryostat with five degrees of freedom and cleaved in UHV using a lever fixed on the sample with silver glue yielding exact planes of cleavage along the van-der-Waals gap.

The measurements were carried out with $\mathrm{HeI}_{\alpha}$ radiation ( $h \nu=21.22 \mathrm{eV}$ ) using an energy analyzer mounted on a two-axis-goniomeler. The energy resolution of the analyzer was $31 \mathrm{meV}$ and the angular acceptance about $\pm 0.5^{\circ}$. The energy scale is fixed to the Fermi level which was determined with an inaccuracy of $\pm 3 \mathrm{meV}$ by measuring the Fermi edge of a thin polycrystalline gold film at $\mathrm{T}=30 \mathrm{~K}$.

First we investigated samples prepared by different excess of Te. In the following we label samples prepared with $1 \mathrm{mg} / \mathrm{cm}^{3}$ and $3 \mathrm{mg} / \mathrm{cm}^{3}$ excess of 'Te as SAMPLE1 and SAMPLE2, respectively. In fig. 4 photoemission spectra of SAMPLE1 at $\mathrm{T}=75 \mathrm{~K}$ are shown. Beginning at $\vartheta=27^{\circ}$ we see a broad structure at $-80 \mathrm{meV}$ binding-energy dispersing to lower binding-energy for smaller $\vartheta$ down to $\vartheta=15^{\circ}$ where the dispersion goes backwards. For angles $\vartheta<10^{\circ}$ the structure disappears. Comparing with fig. 5 where the spectra of SAMPLE2 are plotted we detect in principle the same behaviour for $\vartheta>15^{\circ}$. However, for smaller $\vartheta$ the peak does not move backwards to higher binding-energy and disappears at $\vartheta=11^{\circ}$. These differences are shown in fig. 6 where also the behaviour at room temperature $(\mathrm{T}=300 \mathrm{~K})$ is displayed. Due to the smaller cut-off effects by the Fermi-Dirac distribution the differences become larger. Maxima of the ARPES-weight can be detected especially above the Fermi level. Again at $\mathrm{T}=300 \mathrm{~K}$ SAMPLE2 does not show a backwards directed dispersion for $\vartheta<15^{\circ}$. As the spectra plotted in fig. 4 and fig. 5 are normalized to the same height we have to mention as an additional effect the rapid decrease of the ARPES intensity for $\vartheta<15^{\circ}$. 


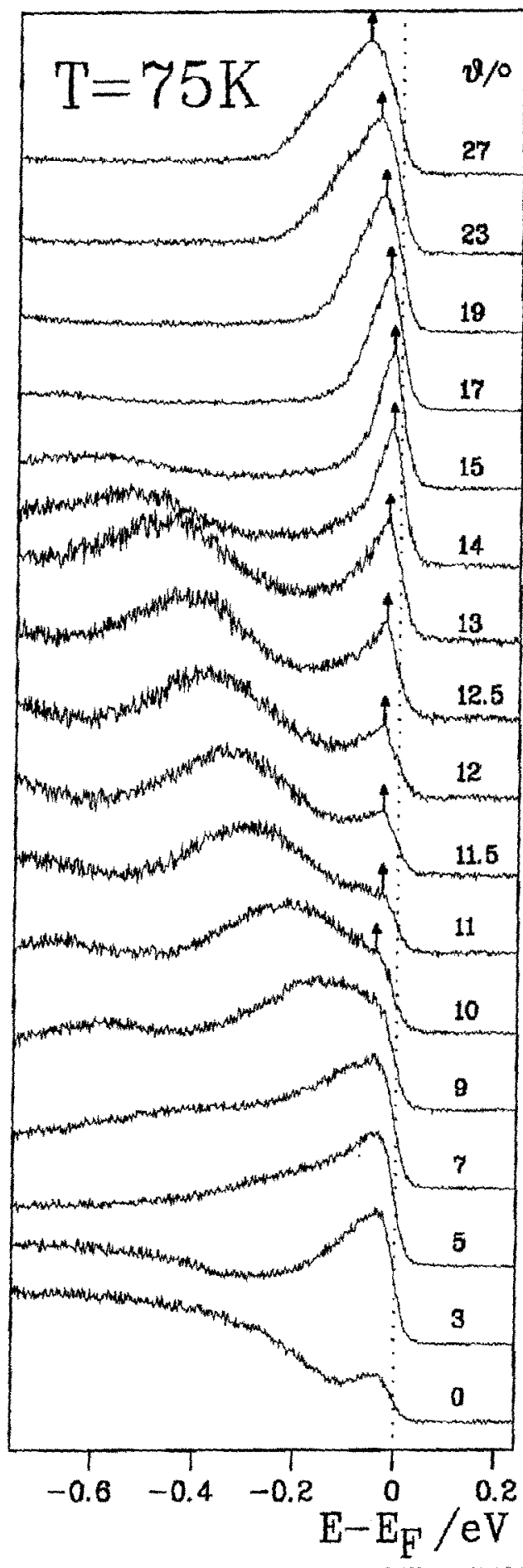

Fig. 4: Photoemission spectra of TiTez (SAMPLE1) along $\widehat{\Gamma M}$ direction taken at $T=75 \mathrm{~K}$ $(\Delta E=38 \mathrm{meV}, h \nu=21.22 \mathrm{eV})$.

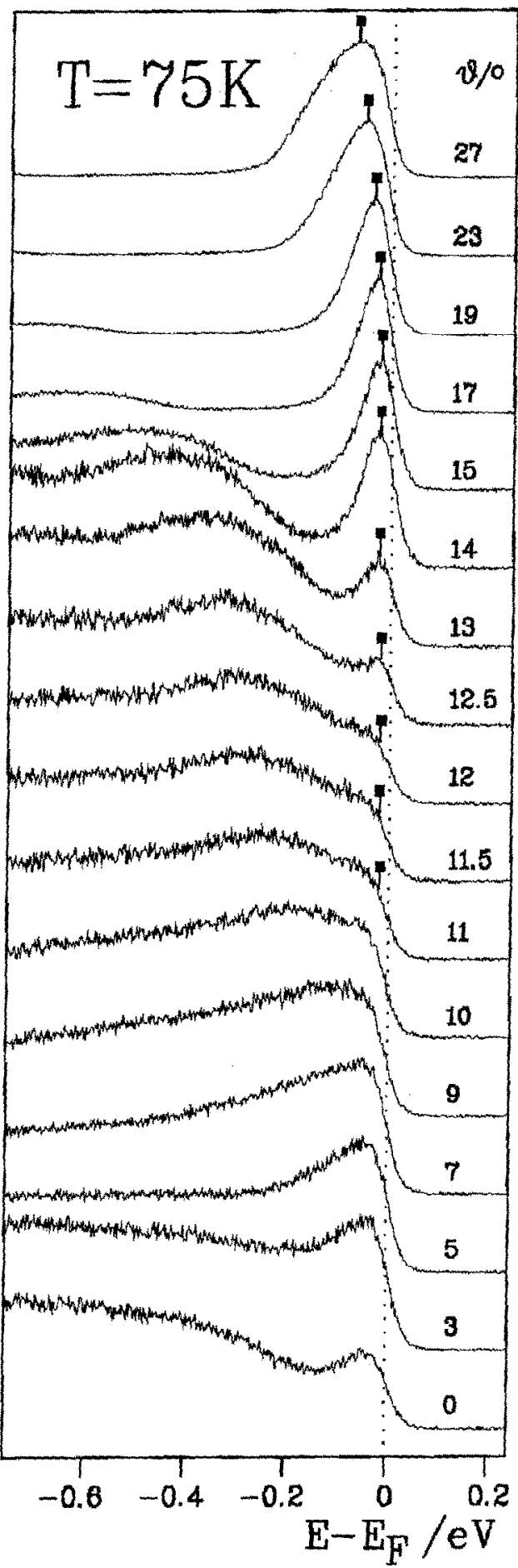

Fig. 5: Photoemission spectra of $\mathrm{TiTe}_{2}$ (SAMPLE2) along $\overline{\Gamma M}$ direction taken at $T=75 \mathrm{~K}$ $(\Delta E=38 \mathrm{meV}, h y=21.22 \mathrm{eV})$. 

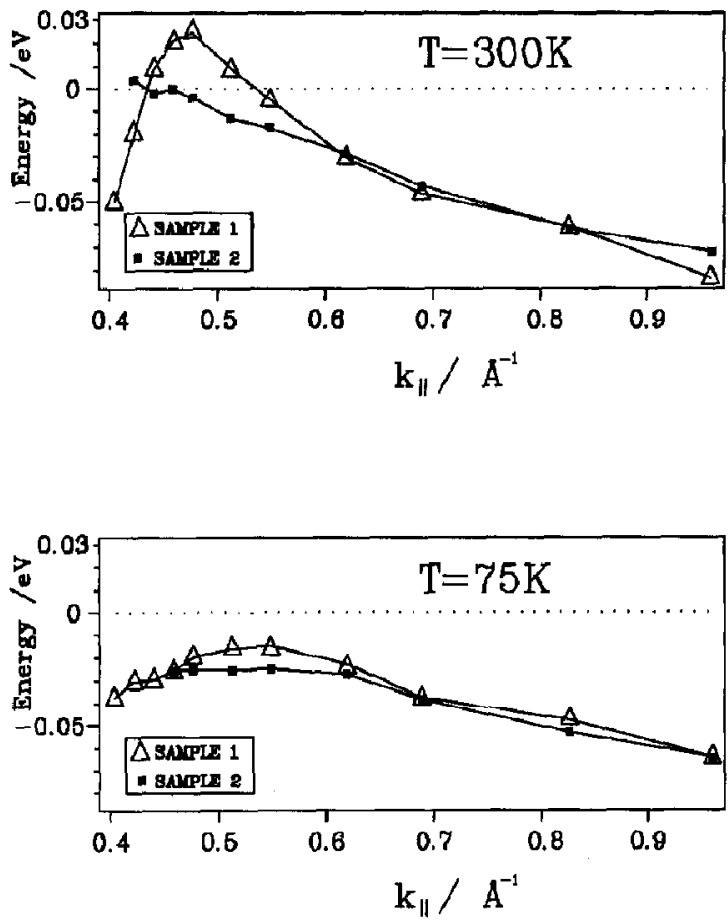

Fig. 6: Positions of ARPES intensity maxima (determined without any fit procedure) of SAMPLE1 and SAMPLE2 at $T=300 \mathrm{~K}$ and $T=75 \mathrm{~K}$. For $T=300 \mathrm{~K}$ there are maxima of spectral intensity located above the Fermi level. The differences between SAMPLE1 and SAMPLE2 concerning dispersion become smaller for decreasing temperatures.

\section{Line Shape Analysis of the Ti-3d $\mathrm{z}^{2}$-Peak}

In a line shape analysis a model function is fitted to the experimental data varying the different fit parameters.

In this paper we look at the two different theoretical model functions mentioned above. The general procedure of the line shape analysis is shown in fig.7. First we have to consider the model-function and the corresponding fit parameters with respect to the physical background. Under these conditions we have three direct fit parameters: the energetical position $\epsilon_{k}^{\prime}$, the renormalised quasiparticle decay constant $\beta^{\prime}$, and an intensity factor $I$.

The model-function is multiplied with the FermiDirac distribution containing as fixed parameters the Fermi energy $E_{F}$ and the temperaturc T which are determined accurately during the measurements.

The angular acceptance of the analyzer is directly connected to the $k_{\|}$-resolution by

$$
\Delta k_{\|}=0.5123 \sqrt{E_{k i n} / e V} \cos \vartheta \Delta \vartheta
$$

The estimated $k_{\|}$resolution manifests itself in an energy resolution due to

$$
\Delta E_{\|}=\frac{\partial E}{\partial k_{\|}} \Delta k_{\|}
$$

where $\partial E / \partial k_{\|}$denotes the energy dispersion of the structure under investigation. Because this factor is obtained as a result of the fit we have to estimate it in a self-consistent way. Taking into account the effect of limited angle resolution we have to integrate the model-function over a range in $\mathbf{k}$-space determined by the factor $\partial E / \partial k_{\|}$. This integration is weighted by the distribution of $\mathrm{k}$ determined by ray-tracing calculations for the electron analyzer. The experimental energy resolution is simulated by convoluting the modelfunction with a gaussian profile of width $\Delta E$.

Finally we have to consider effects which add to the background of the spectra such as inelastic scattering of electrons and satellites due to the He-discharge-lamp. For $\vartheta>15^{\circ}$ the background of the spectra is neglegibly small. For $\vartheta \leq 15^{\circ}$ we subtracted a small Shirley-background [7]. 
1. Fermi-liquid spectral function

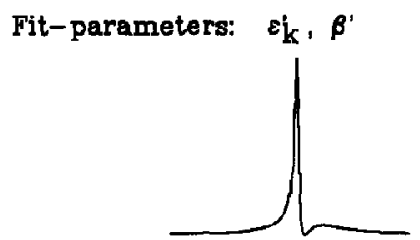

2. Multiplication with Fermi-Dirac distribution

Parameters: $\mathrm{E}_{\mathrm{F}}, \mathrm{T}$ (fixed)

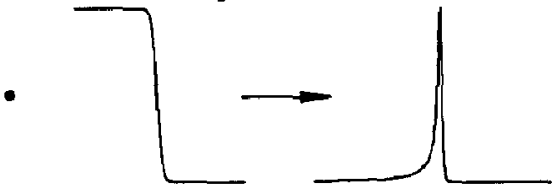

3. Integration in $\mathbf{k}$-space, weighted with electron angle distribution of analyzer. obtained from ray-tracing calculations

Parameter: $\partial \mathrm{E} / \partial \mathbf{k}$ (tixed, self-consistent)

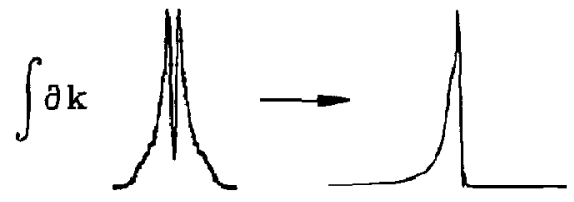

4. Convolution with gaussian-profile (energy resolution of analyzer)

Parameter: $\Delta E$ (fixed)

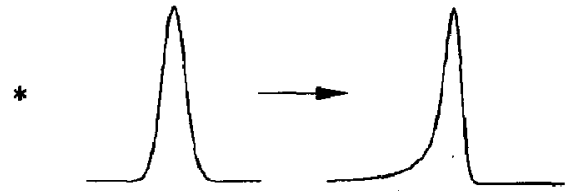

5. Multiplication with intensity factor

Fit-parameter: 1

Fig. 7: Procedure for a Fermi liquid fit.

We use a least-squares-method to accomplish the fit. In fig. 9 the result of a Fermi liquid fit corresponding to the model-function used by Claessen et al. is shown. For $\vartheta<15^{\circ}$ we observe a good agreement between experimental data and the fit. For $\vartheta>15^{\circ}$ the differences between fit and data become larger and above $\vartheta=17^{\circ}$ the model is not suitable to describe the experimental line shape. In fig. 10 the fit corresponding to the model-function proposed by Matho is plotted. The model-parameters $Q$ and $Z_{F}$ were kept constant to 1 and 0.2 , respectively, in order to minimize the number of free fit-parameters. In comparison to fig. 9 there is now a much better agreement between data and fit also for large $\vartheta$. Something special happens at $\vartheta=15^{\circ}$, where the intensity decreases and the band is crossing the Fermi level at room temperature. Looking at the fit-parameter $\epsilon_{k}$ we detect a change of sign, indicating that the main line crosses the Fermi energy. Therefore the fits for $\vartheta<14^{\circ}$ are performed not with the main line, but with the remaining spectral weight in the photoemission region (i.e. $\left.\epsilon<\epsilon_{F}\right)$.

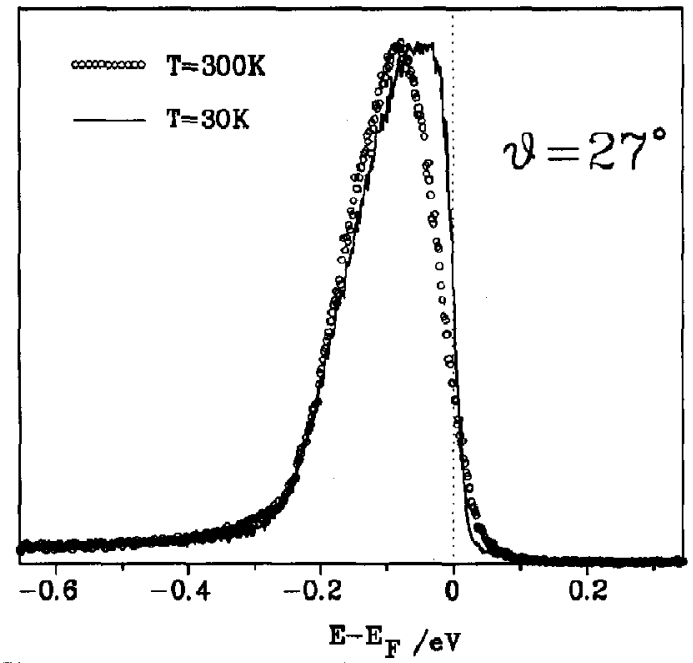

Fig. 8: Comparison of the spectra at the $M$-point $\left(\vartheta=27^{\circ}\right)$ at different temperatures $T=300 \mathrm{~K}$ and $T=30 \mathrm{~K}$ of SAMPLE2 $(\triangle E=31 \mathrm{meV}$, $\mathrm{h} \nu=21.22 \mathrm{eV}$ ).

\begin{tabular}{|c|c|c|c|}
\hline$\vartheta\left[{ }^{0}\right]$ & $\epsilon_{k}^{\prime}[\mathrm{eV}]$ & $\beta^{\prime}\left[\mathrm{eV}^{-1}\right]$ & Amplitude \\
\hline 27 & -87.4 & 18.78 & 0.0136 \\
\hline 23 & -85.4 & 16.94 & 0.0101 \\
\hline 19 & -68.4 & 19.68 & 0.0066 \\
\hline 17 & -50.4 & 16.83 & 0.0057 \\
\hline 16 & -32.0 & 16.65 & 0.0038 \\
\hline 15 & -18.5 & 18.78 & 0.0029 \\
\hline 14 & +19.7 & 17.45 & 0.0033 \\
\hline 13 & +24.6 & 15.66 & 0.0027 \\
\hline 12 & +29.6 & 13.90 & 0.0027 \\
\hline
\end{tabular}

Tab. 1: Parameters corresponding to the fit of fig. 9. 


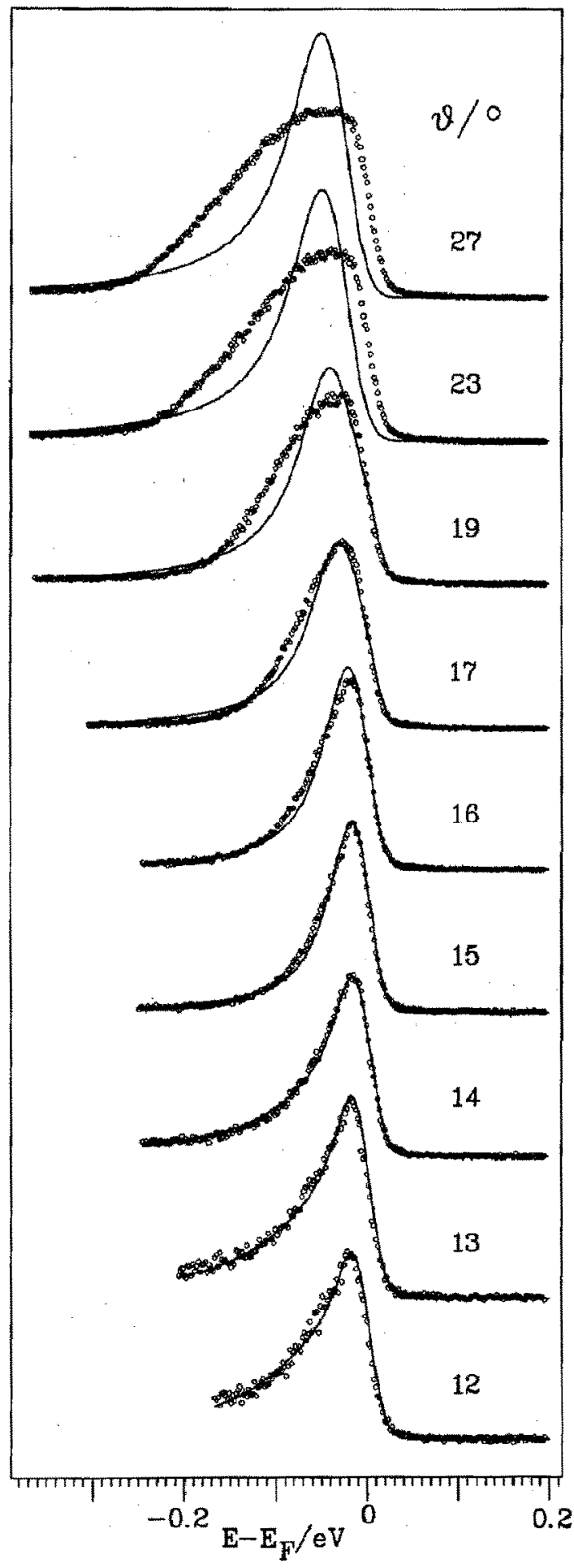

Fig. 9: Fermi liquid fit corresponding to the modelfunction used by Claessen et al. [1] (SAMPLE2, $T=30 \mathrm{~K}$ ).

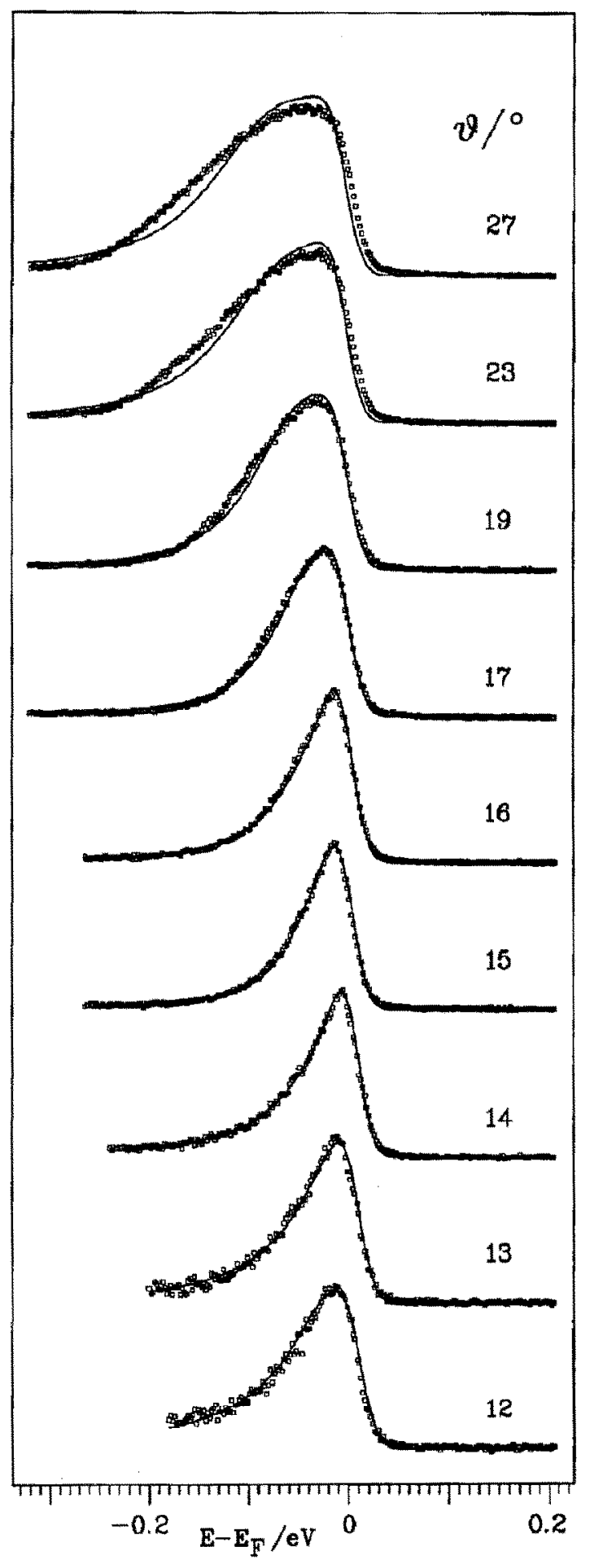

Fig: 10: Fermi liquid fit corresponding to the model-function proposed by Matho [2] (SAMPLE2, T=30K). 
At the zone boundary the fit in fig. 10 still shows some deviations from the experimental data. Fig. 8 shows a comparison of spectra at the $\mathrm{M}$-point taken at $\mathrm{T}=300 \mathrm{~K}$ and $\mathrm{T}=30 \mathrm{~K}$. The energetic position of the $\Lambda$ RPES-maximum is shifted to lower binding-energies. Although the temperature decreases the FWHM of the structure increases due to a broadening at higher bindingenergies. The lower energy tail of the structure is cut off strongly due to the lemperalure dependence of the Fermi-Dirac distribution resulting in an asymmetrical line shape.

\begin{tabular}{|c|c|c|c|}
\hline$\vartheta\left[^{\circ}\right]$ & $\epsilon_{k}[\mathrm{eV}]$ & $\beta\left[\mathrm{eV}^{-1}\right]$ & Amplitude \\
\hline 27 & -79.6 & 252.2 & 0.0331 \\
\hline 23 & -71.0 & 271.6 & 0.0272 \\
\hline 19 & -54.6 & 363.7 & 0.0244 \\
\hline 17 & -40.9 & $42 \overline{4} .4$ & 0.0381 \\
\hline 16 & -28.5 & 402.6 & 0.0414 \\
\hline 15 & -24.8 & $43 \overline{1} .7$ & $0.03 \overline{13}$ \\
\hline 14 & -2.2 & 369.1 & 0.0338 \\
\hline 13 & +4.3 & 383.4 & 0.0246 \\
\hline 12 & +10.9 & 339.1 & 0.0284 \\
\hline
\end{tabular}

Tab. 2: Parameters corresponding to the fit of fig. 10.

\section{Results and Discussion}

Angle resolved photoemission studies on the quasi-two-dimensional, metallic system 1-T$\mathrm{TiTe}_{2}$ demonstrate that ARPES line shapes recorded with high energy and momentum resolution - are able to reflect the nature of an in teracting electron system.

We compared two model-functions describing Fermi liquid behaviour with different low energy expansion of the complex self-energy. Within a tiny interval around the Fermi energy the two model-functions give the same result and are in good agreement with experimental data. Beyond this interval the model-function using the low energy Taylor expansion is not able to reproduce the measurements. In comparison the fit corresponding to the model-function proposed by Matho is distinctly better. Especially for $\vartheta>16^{\circ}$ the deviations of the fit to the experimental data become small.

In agreement with each other both models predict that the $\mathrm{Ti} 3 \mathrm{~d}_{z^{2}}$ derived band crosses the
Fermi level at $\vartheta=14^{\circ}-15^{\circ}$. This is the same point where intensity maxima can be detected above the Fermi energy at room temperature.

An extension of this type of line shape analysis to the photoemission spectra of HTSCs, which are characterized by rather broad and asymmetric peaks and a large apparently k-independent background, depends essentially on the identification of the near- $\mathbf{E}_{F}$ features as of intrinsic nature, which so far has not been unambiguously established. In any case, our Fermi liquid model fit to experimental line profiles of the relatively simple case of $\mathrm{TiTe}_{2}$ demonstrate, how with careful consideration of the underlying many-body physics such a line shape analysis can be successfully performed.

\section{Acknowledgements}

We thank Konrad Matho (CNRS-CRTBT, Grenoble, France) for the comment to paper [1] and further useful suggestions for dealing with Fermi liquid theory. This work is supported by the 'Bundesministerium für Forschung und Technologie' under contract number 055 F KAAB 3.

\section{References}

[1] R. Claessen, R.O. Anderson, J.W. Allen, C.G. Olson, C. Janowitz, S. Harm, M. Kalning, R. Manzke, and M. Skibowski: Phys. Rev. Lett. 69,808 (1992)

[2] K. Matho : private communications (1993)

[3] W. Drube, I. Schäfer, and M. Skibowski: J. Phys, C 20, 4201 (1987)

[4] Y. Koike, M. Okamura, T. Nakanomyo, and T. Fukase: J. Phys. Soc. Jpn. 52, 597 (1982)

[5] J. B. Pendry, in Photoemission and Electronic Properties of Surfaces (Wiley, New York, 1978)

[6] L. Hedin and S. Lundquist: Solid State Physics Vol.23 (Academic Press, New York and London, 1969)

[7] D. A. Shirley Phys. Rev. B 5, 4709 (1972) 